\title{
Ankara bombing: distribution of injury patterns with radiological imaging
}

\author{
Selçuk Parlak ${ }^{A, B, C, D, E, F}$, Muhammed Said Beşler ${ }^{\mathrm{A}, \mathrm{B}, \mathrm{D}, \mathrm{E}, \mathrm{F}}$
}

Ankara City Hospital, Ankara, Turkey

\section{Abstract}

Purpose: To describe and explain the blast injuries and imaging findings in the Ankara terrorist explosion that took place on October 10, 2015.

Material and methods: A total of 77 patients who underwent radiologic imaging were classified as primary, secondary, tertiary, and quaternary, according to the injury type. The patients were evaluated based on body regions, such as head and neck, thorax, abdomen, lower extremity, and upper extremity.

Results: Blast lung injury was identified in one patient and tympanic membrane perforation in seven patients, as the primary injury. Sixty-two of 77 patients had secondary blast injuries caused by shrapnel. The blast injuries were observed in the head and neck (16/77, 20.7\%), thorax (11/77, 14.2\%), abdomen (16/77, 20.7\%), lower extremity $(48 / 77,62.3 \%)$, and upper extremity $(5 / 77,6.4 \%)$. Vascular injuries were seen in eight cases, of which seven were in the lower extremities.

Conclusions: The most common blast injury pattern was of a secondary type in the current study. Lower extremities were the significantly more affected body region, probably due to the bomb exploding at ground level. In mass casualty events, radiologic imaging is located at the centre of patient management.

Key words: computed tomography, radiograph, terrorism, blast injury, Ankara.

\section{Introduction}

Blast injuries previously frequently encountered in wars have begun to affect the civilian population with the increase of domestic or international terrorist bombings in the last few decades $[1,2]$. Penetrating injuries are higher in terrorist attacks than explosions in a military context because civilians have no body armour. Due to the ongoing risk of terrorism, emergency physicians should be familiar with blast injuries [1]. Explosions cause chaos and panic, and in addition, the number of injured victims usually exceeds the capacity of hospitals, unprepared for this uncommon situation [2]. However, it is necessary to direct the injured people to the appropriate departments, to diagnose the life-threatening injuries and start their treatment [3]. The main role of radiology is to provide triage of the victims to the proper sections after fast and accurate imaging $[2,3]$.
Blast injuries are classified as primary, secondary, tertiary, and quaternary. Blast waves are the cause of primary blast injuries (PBIs) in which mostly the affected organs contain gas, such as tympanic membrane and lungs, with the intestines being less frequently affected [4]. The explosion wave due to the pressure difference becomes an air-filled structure and causes PBI while traveling through tissues of varying density. Blast lung, haemothorax, pneumothorax, gastrointestinal perforation, eye rupture, and tympanic membrane perforation are some examples of primary injury [5]. Secondary blast injuries (SBIs) are penetrating injuries resulting from explosive particles or environmental fragments, and they are the most common type of injury in explosion events. Tertiary injuries are blunt, sometimes penetrating injuries caused by the blast wave, propelling the victims into objects, most often seen in the form of fractures. Quaternary injuries are those that can include fire fume inhalation or burns $[1,6,7]$.

\section{Correspondence address:}

Dr. Muhammed Said Beşler, Ankara Numune Research and Education Hospital, 44 Talatpaşa St., 06100, Ankara, Turkey, e-mail: msbesler@gmail.com

Authors' contribution:

A Study design · B Data collection · C Statistical analysis · D Data interpretation · E Manuscript preparation · F Literature search · G Funds collection 
On October 10, 2015 at 10:04 near Ankara railway station, two suicide bombers carried out a terrorist attack on a crowd of people gathering for a meeting. In order to increase the effect of the bombs, it was understood that they had placed ball bearings inside the explosives. In the attack, 102 people were killed and 391 were wounded. Our hospital at that time was one of the most suitable in terms of personnel and technical equipment. Due to the close proximity to the scene and the experience in the field of trauma, most of the injured were brought to the emergency department of our hospital. Radiology, which is an indispensable part of the emergency department, played an important role in the medical management of this unfortunate event. The aim of this study was to evaluate and classify the radiological findings of patients who were injured due to this bomb explosion.

\section{Material and methods}

This current study was approved by the Ethical Committee. After the explosion, the first patient was admitted to the emergency department at 10:34 and the last patient at 17:34. A total of 126 patients were admitted to the emergency department, of whom 77 with radiological imaging findings were included in the study. Patients taken directly to the operation room after admission to the emergency service were excluded from the study. One patient who had sudden cardiac arrest in the tomography room before the examination was also excluded from the study. Cardiopulmonary resuscitation (CPR) was attempted immediately, but the patient died in the CPR room. An emergency radiologist, a resident, and two tomography technicians were on duty. After the explosion, two emergency radiologists, two residents, and two technicians came to the hospital to help their colleagues. X-rays could not be reported because of insufficient time. Verbal information was given to emergency medicine physicians for requested X-rays. All the computed tomography (CT) investigations were reported within an average of three hours, but the surgeons were informed immediately about the patients who needed urgent surgery. For this research, $\mathrm{CT}$ and X-rays of the patients were evaluated retrospectively by two specialist radiologists experienced in emergency radiology.

The patients were divided into primary, secondary, tertiary, and quaternary blast injury groups based on the radiographic and tomographic findings. The injuries were further classified according to the anatomical regions of head and neck, thorax, abdomen, upper extremity, and lower extremity. Both X-rays and CT sections were examined for the presence of shrapnel in superficial and deep soft tissues, bones, and visceral spaces; bone structures were evaluated in detail for fracture; and solid, hollow organs and vascular structures were carefully evaluated for traumatic injury.

\section{Results}

There were 57 men and 20 women in the study group, and their ages ranged from 15 to 75 years (mean 34.55 years). Innumerable metal fragments, ball bearings or millimetric shrapnel were identified on plain radiographs (applied to $73 / 77,94.8 \%$ ) and CT scans (applied to $39 / 77,50.6 \%$ ). Four patients who had no radiograph were investigated with CT. Other diagnostic methods were used: ultrasound in four patients and Doppler ultrasound in five patients. CT angiography was used for diagnosis in eight patients. None of the patients underwent magnetic resonance imaging or digital subtraction angiography that day. The average number of procedures per patient was 1.67.

The results of radiology revealed metallic fragments in 62 patients $(80.5 \%)$. A total of 21 head, four maxillofacial, two orbital, one temporal, three neck, nine cervical spine, 10 chest, four thoracic spine, 11 abdomen, five lumbar spine, three pelvic, six extremity CT procedures, and eight extremity CT angiography studies were performed. Five patients were evaluated with single-pass, whole-body CT examination. In this examination, a non-contrast head CT is performed, and then the area between the skull base and pelvis is scanned after intravascular contrast media. Ten patients had no sign of injury on radiologic examinations. Soft tissue injuries were classified as skin breach or foreign bodies without adjacent bone fractures. Unaccompanied soft tissue injuries were detected in the thorax $(2 / 77,2.6 \%)$, abdomen $(3 / 77,3.9 \%)$, and lower extremities $(25 / 77,32.4 \%)$ (data not shown). Only three patients who were close to the blast point had burn injuries, and no toxic gas inhalation case was encountered. A total of 27 operations, mostly orthopaedic, were performed. Table 1 shows the distribution of injury types among the patients. As shown in Table 2, in the population, the injuries were detected in the head and neck $(16 / 77,20.7 \%)$, thorax $(11 / 77,14.2 \%)$, abdomen $(16 / 77,20.7 \%)$, lower extremity $(48 / 77,62.3 \%)$, and upper extremity $(5 / 77,6.4 \%)$ locations.

False negative reports were inevitable because of the gravity of the mass casualty. The ball bearings and shrap-

Table 1. Distribution of injury types among the patients

\begin{tabular}{|l|c|c|}
\hline $\begin{array}{l}\text { Injury } \\
\text { types }\end{array}$ & $\begin{array}{c}\text { Number of patients } \\
(\%)\end{array}$ & \multicolumn{1}{c|}{ Clinical presentation } \\
\hline Primary & $8(10.3)$ & $\begin{array}{c}\text { Tympanic membrane rupture, } \\
\text { blast lung injury, } \\
\text { intestinal perforation }\end{array}$ \\
\hline Secondary & $62(80.5)$ & $\begin{array}{c}\text { All injuries related } \\
\text { with metallic fragments }\end{array}$ \\
\hline Tertiary & $5(6.5)$ & $\begin{array}{c}\text { Fractures or skin breaches } \\
\text { without being affected } \\
\text { by metallic fragments }\end{array}$ \\
\hline Quaternary & $3(3.9)$ & Burns, toxic gas inhalation \\
\hline
\end{tabular}


nel inside the subcutaneous tissues were not reported in the initial analyse due to their relative insignificance. We noticed ball bearings and shrapnel not reported initially in six patients $(6 / 62,9 \%)$. The prominent faults in the reports pertain to fractures. One metacarpal, one ulnar, two fibular, and one tibial plateau fracture were underdiagnosed (5/27, 18\%). Two ileal perforations were not recognised in the initial evaluation, due to lack of free air and fluid $(2 / 7,28 \%)$. Laparotomy was done and perforations were detected.

The details of the injuries are presented below based on body regions.

\section{Head and neck injuries}

Seven primary-type tympanic membrane perforations were noted. Cerebral and cerebellar injuries were seen in four patients including subarachnoid haemorrhage and parenchymal haemorrhage, with three of them having intracranial ball bearing penetration (Figure 1). Three of the four patients had sustained intraocular injury from the ball bearings in the bomb, with two requiring a total vitrectomy, and the third patient died from life-threatening injuries (Figure 2). Maxillofacial, orbital, mastoid, skull base fractures were seen in six patients, and one had tertiary type orbital and maxillofacial fractures. Only one patient encountered cervical vertebra fracture. Subdural or epidural haematoma was not seen.

\section{Thorax injuries}

In one patient, patchy ground glass appearances in a butterfly pattern were seen due to the primary blast effect (Figure 3). Haemothorax in two patients and a scapula fracture with pneumomediastinum in one patient were identified to have been caused by direct shrapnel damage. A ball bearing shown adjacent to the heart led to second-
Table 2. Distribution of injuries by body regions and number of patients

\begin{tabular}{|c|c|c|}
\hline Body regions & Location or injury type & Number of patients \\
\hline \multirow[t]{6}{*}{ Head and neck } & Brain parenchyma & 4 \\
\hline & Maxillofacial & 4 \\
\hline & Ocular & 4 \\
\hline & Ear & 10 \\
\hline & Neck & 4 \\
\hline & Vertebral fracture & 1 \\
\hline \multirow[t]{5}{*}{ Thorax } & Pneumothorax & 3 \\
\hline & Haemothorax & 3 \\
\hline & Lung parenchyma & 1 \\
\hline & Pneumomediastinum & 1 \\
\hline & Fracture & 2 \\
\hline \multirow[t]{7}{*}{ Abdomen } & Liver & 2 \\
\hline & Kidney & 2 \\
\hline & Pancreas & 1 \\
\hline & Uterus & 1 \\
\hline & Intestinal perforation & 7 \\
\hline & Fracture & 5 \\
\hline & Vascular & 1 \\
\hline Upper extremity & Fracture & 5 \\
\hline \multirow[t]{2}{*}{ Lower extremity } & Fracture & 22 \\
\hline & Vascular & 7 \\
\hline
\end{tabular}

ary type pneumothorax (Figure 4). Haemopneumothorax was detected in one patient as an SBI. Sternal and costal fractures were observed in only two patients.

\section{Abdomen injuries}

Sixteen patients had injuries to the abdominal region, all of which were SBI. Gastrointestinal system perforations

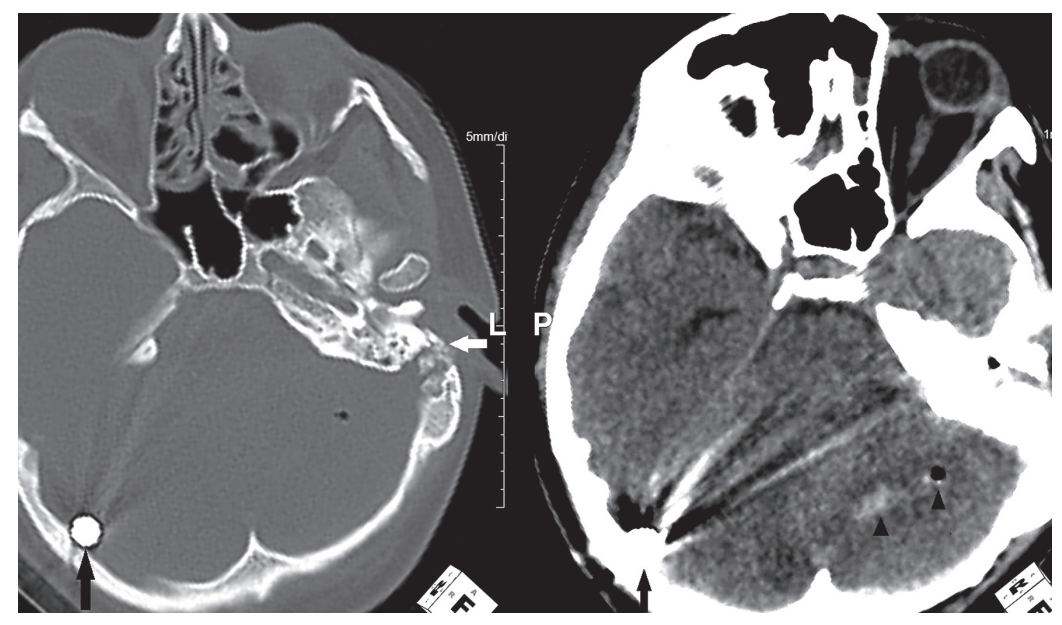

Figure 1. Non-contrast computed tomography of the head in the bone and parenchyma window. A ball bearing is seen adjacent to the internal tabula of the occipital bone (black arrows). Haemorrhage and air density are present in the left cerebellar hemisphere (arrowheads). The left temporal bone fracture indicates the entry of the ball bearing (white arrow) 


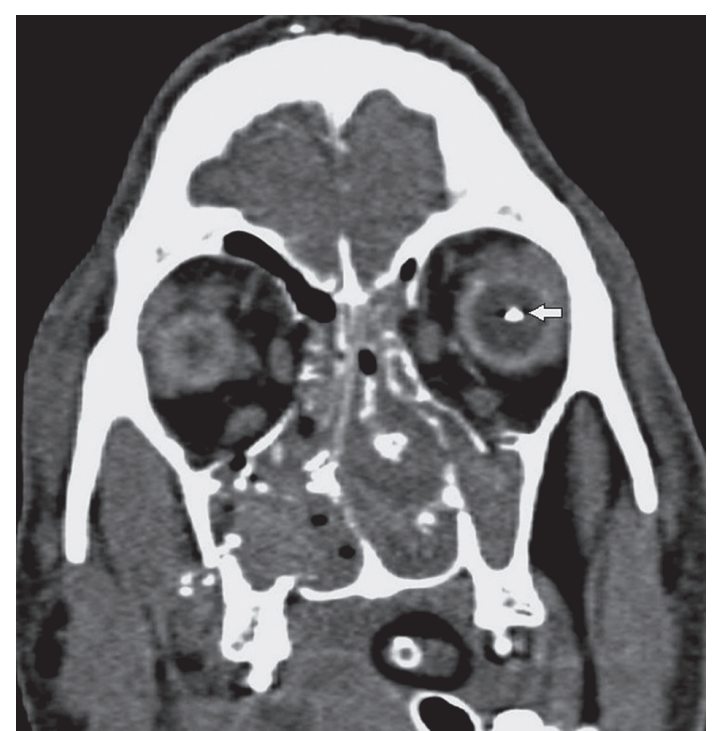

Figure 2. A shrapnel injury to the left globe (arrow). The presence of multiple maxillofacial fractures is noted on the reformatted coronal computed tomography image. Maxillary sinuses and nasal cavity are obliterated with haematoma

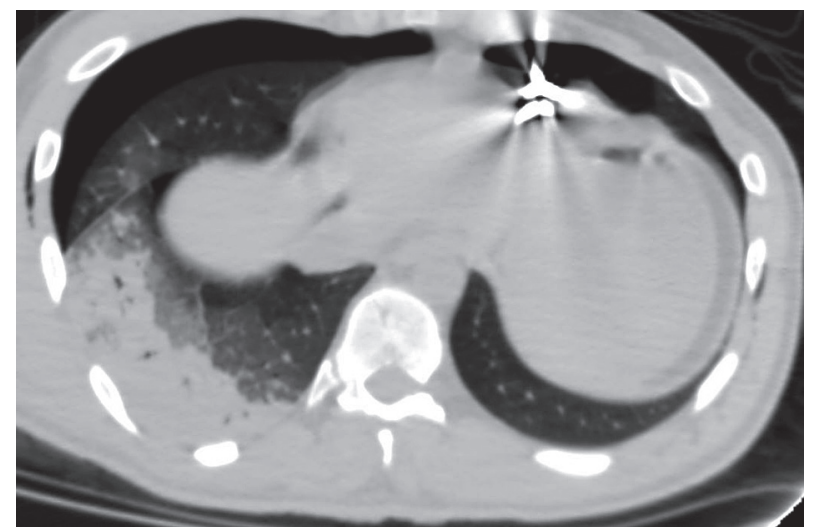

Figure 4. A secondary blast injury on an axial thoracic computed tomography image, revealing right-sided pneumothorax accompanying pulmonary contusion caused by a metallic shrapnel fragment located next to the heart

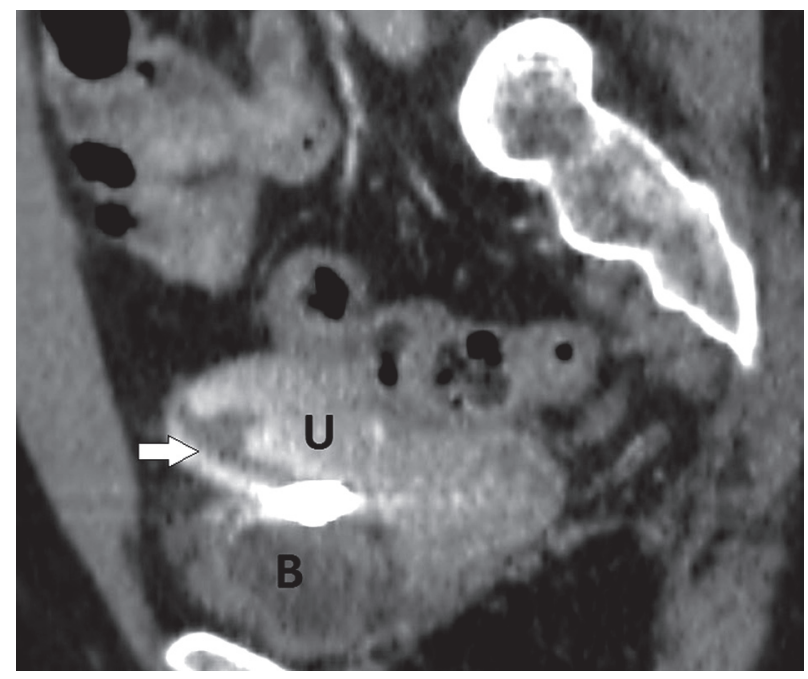

Figure 6. The reformatted sagittal computed tomography image of a 41-yearold female patient, showing a shrapnel fragment (arrow) causing laceration of the uterus ( $\mathrm{U}$ - uterus, $\mathrm{B}$ - bladder)

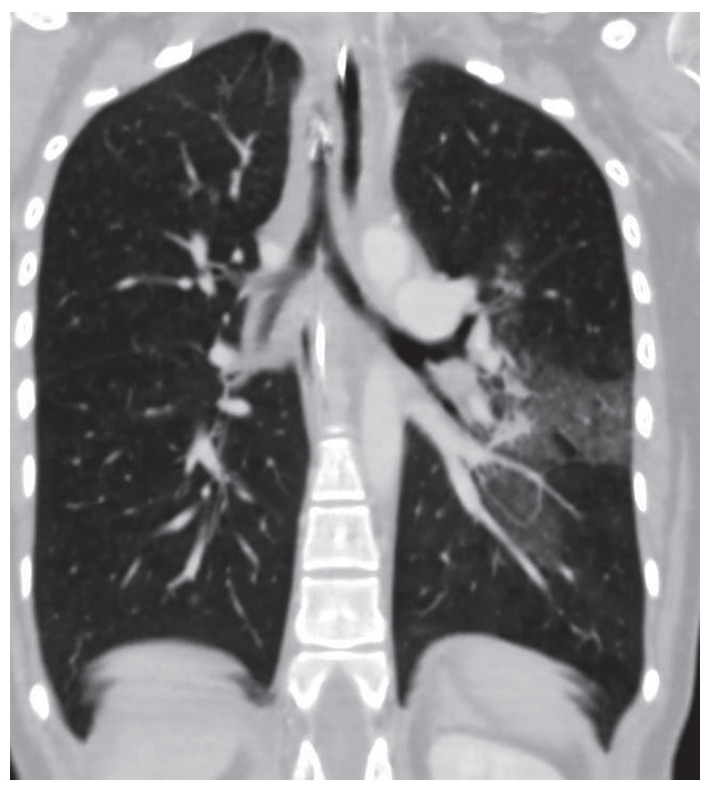

Figure 3. An example of a primary blast injury to the lung. The thoracic computed tomography demonstrates ground glass opacities in the left perihilar region compatible with butterfly pattern. No ball bearing, fracture, or soft tissue injury was detected in this victim

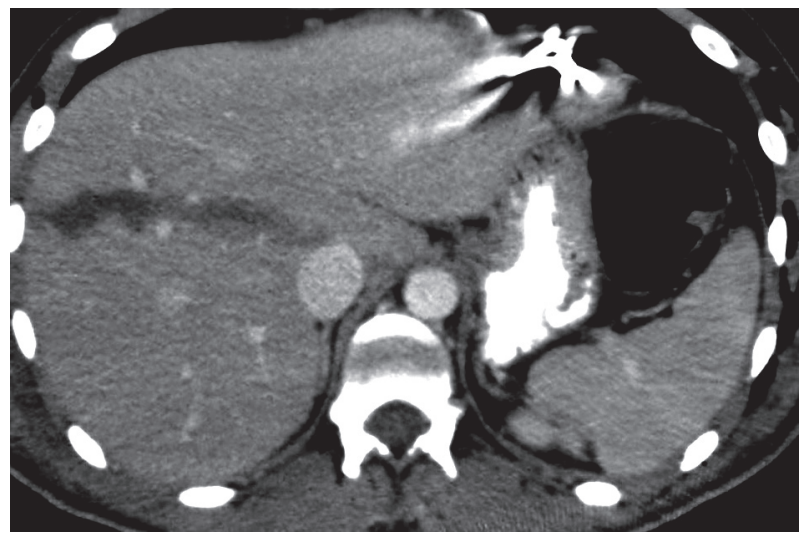

Figure 5. The abdominal computed tomography image of the patient in Figure 4, revealing a hypodense line compatible with a grade 3 laceration in the right lobe of the liver

involving the ileum or colon were seen in seven patients, for whom surgery was undertaken for resection and repair. No primary bowel perforation was found in the study population. One patient had simultaneous liver, kidney, pancreas, and stomach injuries. One further patient had liver and kidney laceration (Figure 5). Uterine perforation caused by the ball bearing penetration was examined in one patient (Figure 6). Internal iliac vein damage was observed in one patient as abdominal vascular injury. Lumbar vertebra, iliac bone, and sacrum fractures were observed in five patients.

\section{Extremity injuries}

The types of extremity injuries were predominantly of the secondary type with a few tertiary blast wave type injuries. 

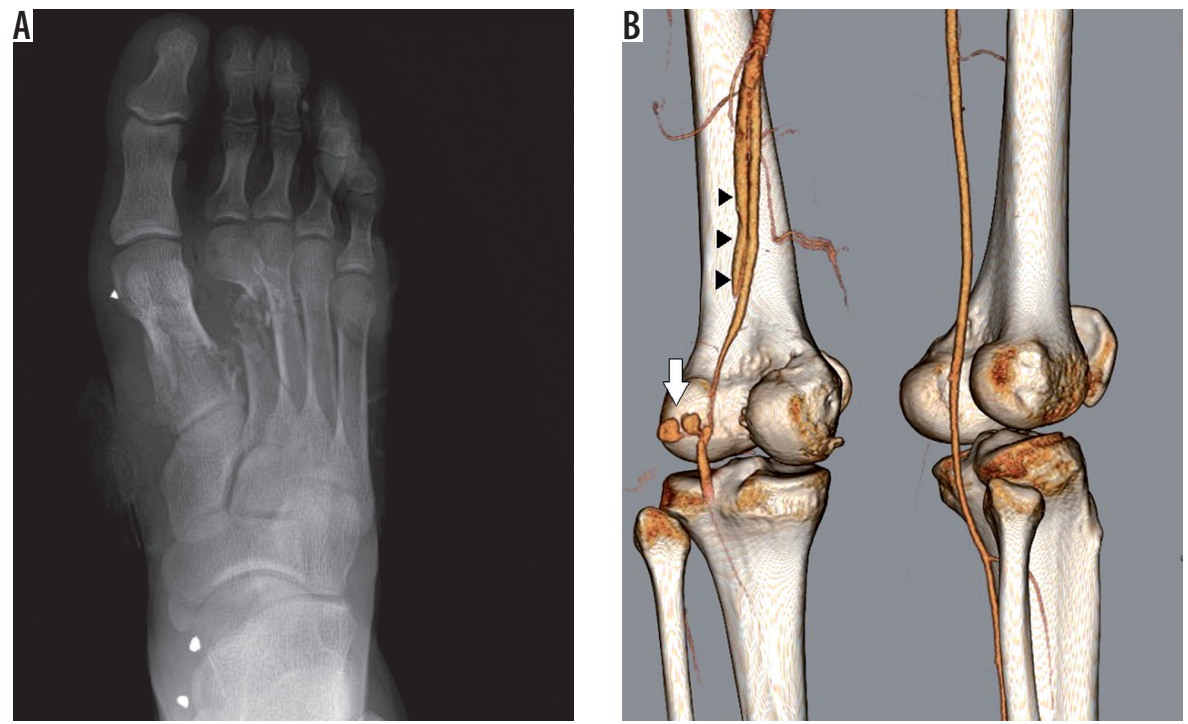

Figure 7. A right foot $X$-ray showing fragmented fractures in the first and second metatarsal bones and a fracture in the third metatarsal bone. The image reveals shrapnel fragments located in the soft tissues in the foot and ankle (A). A three-dimensional computed tomography angiography image showing traumatic pseudoaneurysms (white arrow) in the left popliteal artery. The popliteal artery is occluded before trifurcation and there was no opaque filling. Opaque filling was prominent in the femoral vein, compatible with an arteriovenous fistula (black arrowheads) (B)

Four patients had tertiary type injuries involving lower extremity fractures and soft tissue injuries. Five patients had upper extremity injuries consisting of one humeral, one radial, one metacarpal, and two ulnar fractures; however, the vascular structures of the patients were intact. Metallic fragments were mainly detected in lower extremities. Twenty-two patients had lower extremity fractures mostly consisting of tibial $(14 / 22,63.6 \%)$, followed by talar $(1 / 22$, $4.5 \%)$, metatarsal $(2 / 22,9 \%)$, femoral $(7 / 22,31.8 \%)$, and fibular (9/22, 40.9\%) (Figure 7A). Seven patients had peripheral vascular damage affecting the superficial femoral artery, popliteal artery, and common femoral vein; however, two patients underwent lower extremity amputation. The popliteal artery was the most affected vascular structure in which pseudoaneurysm and arteriovenous fistula were also detected (Figure 7B).

\section{Discussion}

Terrorist bombing attacks have increased in recent years, and a new pattern of terrorism targets masses, not individuals $[1,8]$. In the process after the Ankara bombing on October 10, 2015, two more terrorist bomb attacks were carried out targeting civilian populations. Thus, medical personnel, especially those working in places prone to terrorism, have had to learn about the particular injury patterns that result from these attacks [3,5].

In a bomb attack, the explosive substance transforms from a solid to gaseous state, creating the blast wave, which affects nearby objects and living creatures. Blast waves are rapidly progressive [7,9], displace the surrounding air, and creating a wind reaching several hundred kilometres per hour, which can result in shear forces that may cause limb amputations [3].
Blast injuries are classified as primary, secondary, tertiary, and quaternary; however, in a bomb attack, people usually have multiple injuries, with two or more injury patterns being combined in the same victim [1,5]. Five of our patients had both primary and secondary injury, and one patient had primary, secondary, and tertiary injury. One of the quaternary injured victims also had a secondary injury. If an individual is close enough to the point of detonation, the initial blast wave increases pressure in the body, causing stress and shear, particularly in gas-filled organs, such as the ears, lungs, and possibly but rarely the bowels. Blast injuries can include pneumothorax, haemothorax, pulmonary contusion, traumatic emphysema, and air embolism. Traveling at high speed, shrapnel fragments cause multiple penetrating injuries after the explosion. These secondary blast injuries are the most common cause of explosion-related injury $[6,7,9,10]$. Singh et al. investigated the Boston Marathon bombing and detected shrapnel in 32 of the 43 patients [6]. SBIs were the majority of the injuries in the current study group, too. Shrapnel was detected in all these patients by X-ray or CT. SBIs were detected in $62 / 77(80.5 \%)$ of the victims. In the literature review, SBI dominancy was found in open-space bombings $[5,6,11,12]$. The blast wind also propels people onto hard surfaces, causing blunt or tertiary injuries generally manifested as fractures $[6,7,9]$.

Severe head trauma is one of the most common causes of death in blast patients [2]. Injuries can be of four types, but they are mostly secondary type injuries. Intracranial haemorrhages are examples of secondary injuries, while examples of tertiary injury due to blunt effect are soft tissue injuries and fractures [10]. We identified 16 head and neck injured patients. In most cases $(10 / 16,62.5 \%)$, the cause was the ball bearings placed in the bomb to increase 
the effect of the explosion. Three of the four patients with cerebral and cerebellar haemorrhage had intracranial ball bearings. CT is considered as the gold standard technique to evaluate the blast injured eye, particularly for penetrating trauma [1]. Primary injuries result from shear forces and manifest as haemorrhages, detachments, or globe rupture. Blast-induced projectile fragments cause secondary injuries, which are more common than primary ones, and most are foreign bodies, globe rupture, and orbital fractures $[13,14]$. Two of the three patients with intraocular ball bearings underwent total vitrectomy; however, the other patient died on the same day from other injuries.

One of the major causes of immediate death in victims is blast lung injury. The proximity of the person to the explosion point and the bombing being in a closed area increases the incidence and severity of primary blast lung injury [2]. This type of injury was reported in $0.6 \%$ to $8.4 \%$ of blast explosions [1]. This rate was higher in victims who died after the explosion (17\% to $47 \%$ ) [5]. However, early diagnosis and aggressive treatment reduce the mortality rate in these patients. Hirshberg et al. followed 11 patients with primary blast lung injury for one year and found that the lungs had completely healed [15]. We evaluated a case of blast lung injury with patchy ground glass appearances in the current study. Radiologically, primary blast lung injury presents with pulmonary infiltrates, more commonly bilateral and diffuse with a typical butterfly distribution. A primary blast wave lung injury can also cause pneumothorax, haemothorax, bronchopleural fistula, and air embolism [2].

In the literature, abdominal injury frequency varies between $5 \%$ and $12 \%$ in bomb blast cases $[2,16]$. Abdominal organ injuries are mostly due to secondary type blast effects, followed by tertiary types $[2,5]$. The blast wave may cause oedema, haemorrhage, contusion, intramural haematoma, and rupture in the bowel wall in the case of PBIs [1]. Singh et al. investigated abdominal injuries in the Boston Marathon bombing and found that 11 patients had positive findings. Most of the cases had shrapnel in superficial tissues, but no penetration of the peritoneum was seen in any of the patients. Singh et al. suggested that the absence of visceral injury in the abdomen is due to the low intensity of the explosion [16]. We found abdominal injury in 16 of the 77 patients (20.7\%). All of these were SBIs, and no PBI was detected. The reason for there being no abdominal PBIs in the Boston and Ankara bombings may be the result of the explosions taking place in an open area. However, in the current study, there were seven intestinal perforations, all of which were evaluated as secondary. Unlike the bombings of Boston and Ankara, the explosion in a closed space in the Madrid train attack led to primary bowel perforations [17]. As a unique example, not seen in the literature, a uterine perforation due to ball bearing in a patient was encountered in the current study.

Because explosive devices are usually left on the ground, lower extremity injuries were the most common in mass casualty events [7]. Studies of blast victims in Israel, Iraq, Pakistan, and Lebanon showed an incidence of lower extremity injuries in $17-39 \%[4,18]$. Lower extremities were also found to be the most affected body region in the Boston Marathon explosion (37\%); 41 of 43 patients (95\%) sustained SBIs to the lower extremities with some combination of retained shrapnel, soft tissue lacerations without retained shrapnel, and lower extremity amputation. Eight patients (20\%) had lower extremity fractures, and five patients (12\%) had vascular injuries involving lower extremity arteries. Ten of the 43 patients (23\%) sustained lower extremity amputations [4]. In the current study, as in the literature, the most injured body regions were lower extremities (48/77, 62.3\%). Lower extremity bone fractures were detected in 22 patients. In total, two patients underwent amputation. Vascular injury was diagnosed in seven patients by using CT angiography. The most affected artery was the popliteal artery. These seven patients underwent vascular repair surgery, and two were amputated below the knee. In the study by Yazgan and Aksu, of the 28 people brought to the hospital after the explosion in Ankara, the most common injury site was the torso, with the lower extremity injuries being fewer [11]. This may be because the more severe cases, including extremity injury, were admitted to our hospital, which was the nearest centre to the scene.

The blast location affects the impact of the explosion, i.e. if it is in an open space or closed space. In closed areas, more pressure differences occur and more environmental fragments fly around. The rates of PBIs, SBIs, and mortality are also high in closed-area attacks $[1,2,9]$. PBI cases and immediate death related with PBIs occur more often in closed-space detonations. Of all the explosions in Iraq and Afghanistan, most (95\%) occurred in open spaces, and most injuries and deaths were caused by fragments [9]. In the 2015 Ankara explosion, most of the injuries were of secondary type, in accordance with other open-air explosions.

There are some limitations to our study. Most of the victims were brought to our hospital; however, some had to be transferred to five different hospitals. The patients who were taken directly to the operation room without radiological examination could not be included in the study, most of whom were severely injured with SBIs. In addition, the CT images of a small number of patients could not be loaded into the picture archiving and communication system (PACS) due to the intensity of the workload on that day; thus, a few patients were not included in the study.

\section{Conclusions}

The types of explosion once encountered in military conflicts have become targeted at civilians in recent years. Unfortunately, these types of injuries have become a familiar situation, especially for emergency physicians and emer- 
gency radiologists who work in terror zones. The first stage of treatment in these patients is an accurate diagnosis, with radiology playing an important role in this process. The most useful imaging method utilised after the Ankara explosion was CT. The most common type of injuries were SBIs, and the most common cause was ball bearings detected in superficial and deep tissues on CT and X-ray. In our study, the most common site of injury was the lower extremities, probably due to the bomb exploding at ground level. Familiarity with injury patterns and imaging findings in mass casualty events can lead to correct diagnoses and help to reduce mortality and morbidity.

\section{Conflict of interest}

The authors report no conflict of interest.

\section{References}

1. Mathews ZR, Koyfman A. Blast injuries. J Emerg Med 2015; 49: 573-587.

2. Hare SS, Goddard I, Ward P, et al. The radiological management of bomb blast injury. Clin Radiol 2007; 62: 1-9.

3. Sosna J, Sella T, Shaham D, et al. Facing the new threats of terrorism: radiologists' perspectives based on experience in Israel. Radiology 2005; 237: 28-36.

4. Konwinski RR, Singh A, Soto J. Imaging of lower extremity trauma from Boston Marathon bombing. Emerg Radiol 2016; 23: 433-437.

5. Wolf SJ, Bebarta VS, Bonnett CJ, et al. Blast injuries. Lancet 2009; 374: 405-415.

6. Singh AK, Goralnick E, Velmahos G, et al. Radiologic features of injuries from the Boston Marathon bombing at three hospitals. AJR Am J Roentgenol 2014; 203: 235-239.

7. Singh AK, Ditkofsky NG, York JD, et al. Blast injuries: from improvised explosive device blasts to the Boston Marathon bombing. Radiographics 2016; 36: 295-307.

8. Khorram-Manesh A. Europe on fire; medical management of terror attacks - new era and new considerations. Bull Emerg Trauma 2016; 4: $183-185$

9. Champion HR, Holcomb JB, Young LA. Injuries from explosions: physics, biophysics, pathology, and required research focus. J Trauma 2009; 66: 1468-1477.

10. Singh AK, Buch K, Sung E, et al. Head and neck injuries from the Boston Marathon bombing at four hospitals. Emerg Radiol 2015; 22 : 527-532. https://doi.org/ 10.1007/s10140-015-1322-9.
11. Yazgan C, Aksu NM. Imaging features of blast injuries: experience from 2015 Ankara bombing in Turkey. Br J Radiol 2016; 89(1062):20160063. https://doi.org/10.1259/bjr.20160063.

12. Brunner J, Singh AK, Rocha T, et al. Terrorist bombings: foreign bodies from the Boston Marathon bombing. Semin Ultrasound CT MR 2015; 36: 68-72.

13. Ritchie JV, Horne ST, Perry J, et al. Ultrasound triage of ocular blast injury in the military emergency department. Mil Med 2012; 177: 174-178.

14. Morley MG, Nguyen JK, Heier JS, et al. Blast eye injuries: a review for first responders. Disaster Med Public Health Prep 2010; 4: 154-160.

15. Hirshberg B, Oppenheim-Eden A, Pizov R, et al. Recovery from blast lung injury: one-year follow-up. Chest 1999; 116: 1683-1688.

16. Singh AK, Sodickson A, Abujudeh H. Imaging of abdominal and pelvic injuries from the Boston Marathon bombing. Emerg Radiol 2016; 23: 35-39.

17. Turegano-Fuentes F, Caba-Doussoux P, Jover-Navalon JM, et al. Injury patterns from major urban terrorist bombings in trains: the Madrid experience. World J Surg 2008; 32: 1168-1175.

18. Mirza FH, Parhyar HA, Tirmizi SZ. Rising threat of terrorist bomb blasts in Karachi - a 5-year study. J Forensic Leg Med 2013; 20: 747-751. 